\title{
Utilisation des indices diatomiques pour la mesure de la qualité des eaux du bassin Artois-Picardie : bilan et perspectives
}

\author{
J. Prygiel ${ }^{1}$ \\ M. Coste 2
}

Mots clés : Diatomées, indices diatomiques, cours d'eau, monitoring, pollution, bassin Artois-Picardie.

En raison de la nature particulière des cours d'eau de son bassin, l'Agence de l'Eau Artois-Picardie a adopté en 1988 les diatomées et les indices diatomiques pour l'estimation de la qualité biologique de l'eau. Six indices ont été testés. Les résultats montrent que l'IPS ou Indice de Polluosensibilité Spécifique (Coste in CEMAGREF 1982) est le seul à donner une évaluation réaliste de la qualité des eaux. Cet indice intègre en effet des phénomènes tels que la pollution organique exprimée par la $\mathrm{DBO}_{5}$, la DCO ou l'ammonium, la salinité exprimée par la conductivité et les chlorures ou encore l'eutrophisation qu'elle soit réelle et exprimée par la chlorophylle ou potentielle et exprimée par les phosphates. Ces trois phénomènes sont les principaux facteurs responsables de la variation de la qualité des eaux dans le bassin. Par ailleurs, les conditions physiocochimiques étant particulièrement stables tout au long de l'année, les indices diatomiques calculés en septembre sont représentatifs de là qualité estivale des eaux. Différentes simulations ont été effectuées, à l'aide du logiciel OMNIDIA, en vue de la mise au point d'un indice pratique fiable applicable en routine. Les résultats montrent que la meilleure approche consisterait à travailler au niveau générique avec parfois des déterminations à l'espèce.

\section{The use of diatom indices for measuring water quality in the Artois-Picardie basin}

Keywords : Diatoms, diatom indices, water-course, control, pollution, Artois-Picardie basin.

Because of the special nature of the streams in its basin, the Water Agency of Artois-Picardie adopted in 1988 the diatoms and diatom indices for the assessment of water quality. Six indices have been tested. The results show that the IPS or Specific Polluosensitivity Index (Coste in CEMAGREF 1982) is the only on provide a realistic assessment of water quality. This index integrates three variables : organic pollution expressed as $\mathrm{DBO}_{5}, \mathrm{DCO}$ or ammonium, salinity expressed as conductivity and chlorides, and eutrophication which, when actual, is expressed as chlorophyll levels and, when potential, is expressed as phosphate levels. These three variables ăre the principal factors responsible for variations in water quality within the basin. In addition, the physicochemical conditions were markedly stable throughout the year and therefore the diatom indices calculated in September are representative of summer water quality. Different simulations have been made, using the program OMNIDIA, in order to determine a practical index that can be used routinely. The results show that the best approach is to work at the generic level with occasional determinations to species.

\section{Introduction}

Les macroinvertébrés sont les organismes les plus utilisés pour l'estimation de la qualité des eaux continentales, șaumâtres et marines (Blandin 1986).

\footnotetext{
1.Agence de l'eau Artois-Picardie, 764 boulevard Lahure, 59508 Douai cedex, France.

Laboratoire d'écologie numérique, SN3, UST Lille, 59650 Villeneuve d'Asq cedex, France.

2. CEMAGREF Bordeaux, 50 avenue de Verdun, Gazinet, 33610 Cestas, France.

Texte d'une communication effectuée dans le cadre du Colloque - Limnologie appliquée et application de la Limnologie Besançon, Franche-Comté, 16-19 Novembre 1992.
}

Divers indices de qualité basés sur les macroinvertébrés ont été proposés depuis les années 60 (Woodiwiss 1964 ; Chandler 1970 ; De Pauw \& Vanhooren 1983 ; Verneaux \& Tuffery, 1967, Verneaux et al. 1982, Wright et al. 1988) et sont largement utilisés pour le suivi des réseaux de surveillance nationaux (Newman 1988). Parmi les autres organismes largement utilisés se trouvent les algues et plus particulièrement les diatomées. Ces dernières sont considérées comme faisant partie des meilleurs bioindicateurs (Schoeman \& Haworth 1986, Round 1991, Cox 1991) et de nombreuses méthodes ont été proposées (Patrick 1949, Vanlandingham 1976, Lange-Bertalot 1979, Van Dam 1982, Slàdeček 1986, 
Steinberg \& Schiefele 1988). Leur utilisation demeure cependant relativement limitée (Whitton et al. 1991), particulièrement en France (Coste et al. 1991). Plusieurs raisons ont été avancées pour expliquer ce faible intérêt : Les difficultés d'identification qui requièrent de bonnes connaissances taxonomiques, la nécessité de distinguer les cellules vivantes des cellules mortes (Schoeman \& Haworth 1986), la faible capacité intégratrice et la nécessité d'un échantillonnage quantitatif pour l'appréhension d'effets toxiques (Descy \& Coste 1989). Concernant les indices diatomiques, certains auteurs leur reprochent leur subjectivité (Rott 1991), leur formulation mathématique (Sullivan 1986) et les risques liés à une utilisation abusive (Fabri 1987). Cox (1991) estime que les indices sont basés sur un nombre trop limité de paramètres et critique l'utilisation d'abondances relatives. En fait, il apparait que le principal obstacle à une plus large utilisation des indices diatomiques et des diatomées en général, est l'absence de standardisation (Cazaubon 1991, Friedrich 1991) mais aussi l'absence d'une méthodologie pratique et largement accessible. Depuis les années 70 , Coste $(1976,1978)$ sur la Seine, Descy (1979) dans la partie belge de la Sambre et de la Meuse, Fabri \& Leclercq (1986) et Leclercq \& Depiereux (1987) sur les cours d'eau des Ardennes belges ont proposé divers systèmes de classification des espèces selon leur sensibilité globale à la pollution. Dans la plupart des cas, ces ordinations réalisées par analyses multivariables ont conduit à la définition d'indices diatomiques. En raison de la nature de ses cours d'eau et de la spécificité des problèmes de pollution, l'Agence de l'Eau Artois-Picardie a décidé d'utiliser les diatomées et les indices diatomiques en complément aux indices macroinvertébrés, pour la surveillance biologique de son réseau dès 1988 (Prygiel 1991). Divers indices ont ainsi été testés et une carte de qualité des eaux du bassin ArtoisPicardie a été éditée (A.E.A.P. 1992). L'utilisation de ces. indices n'est cependant pas sans poser de problèmes. La plupart d'entre eux nécessitent des identifications à l'espèce et demeurent par conséquent difficiles à mettre en œuvre par des services techniques chargés du suivi de la qualité des eaux. Le but de cette étude est double : d'une part, présenter les principaux résultats comparatifs des performances de plusieurs indices par rapport aux caractéristiques chimiques des eaux du bassin ; d'autre part étudier quelques pistes en vue de proposer une méthodologie pratique applicable en routine. Sont successivement envisagés la réduction de l'effectif à compter, la limitation dës investigations aux espèces dominantes de l'échantillon et une identification au genre avec dans certains cas une identification à l'espèce.

\section{Matériel et méthodes}

La majeure partie du réseau de surveillance du Bassin Artois-Picardie a été étudiée de mai et septembre 1990. 355 prélèvements ont ainsi été réalisés. A chaque site, les principaux paramètres chimiques ont été mesurés. Les différentes formes d'azote et de phosphore sont respectivement exprimées en nitrate, nitrite, ammonium, azote Kjeldhal et en orthophosphate. $\mathrm{La} \mathrm{DBO}_{5}$ et la DCO (demandes biochimique et chimique en oxygène) sont exprimées en $\mathrm{mg} \mathrm{d}^{\prime} \mathrm{O}_{2}$ par litre. Dans le même temps, les diatomées ont été prélevées par grattage de substrats naturels (cailloux, palplanches des canaux...) sur une surface d'environ $10 \mathrm{~cm}^{2}$. A défaut, des prélèvements ont été réalisés sur substrat végétal. Après nettoyage à l'eau oxygénée concentrée, les diatomées sont montées dans du naphrax (IR $=1,74)$ pour l'observation au microscope au grossissement 1000 . Chaque préparation a fait l'objet d'un comptage de 400 valves minimum. Les déterminations ont été faites au niveau spécifique voire infraspécifique selon Krammer \& Lange-Bertalot (1986-1991) et Round et al. (1990). Les inventaires ont été saisis sur le logiciel OMNIDIA (Lecointe et al. 1993) qui permet le calcul de 6 indices diatomiques : L'Indice de Polluosensibilité Spécifique ou IPS (Coste in CEMAGREF 1982) ; l'indice de Descy ou DES (1979) ; l'indice de Slàdeček (1986) ou SLA ; l'indice de Leclercq \& Maquet (1987) ou ILM ; l'indice Diatomique Générique ou IDG (Coste \& Ayphassorho 1991) et enfin l'indice CEC (Descy \& Coste 1991). Les 5 premiers sont calculés selon la formule proposée par Zelinka \& Marvan (1961) : ID $=\Sigma A_{j} v_{j} i_{j} / \Sigma A_{j} v_{j}$ où $A_{j}$ est l'abondance relative de l'espèce $j, v_{j}$ est la valeur indicatrice de l'espèce $\mathrm{j}(1 \leqslant \mathrm{~V} \leqslant 3)$ et $\mathrm{i}_{\mathrm{j}}$ est la sensibilité à la pollution de l'espèce $\mathrm{j}(1 \leqslant$ $\mathrm{i} \leqslant 5$ ). L'indice CEC est calculé à partir d'une grille à double entrée où les groupes horizontaux sont composés d'espèces à large distribution rangées par ordre de tolérance croissante à la pollution et les groupes verticaux d'espèces à faible amplitude écologique classées par ordre d'apparition le long d'un écosystème théorique amont-aval. Ces indices utilisent un jeu d'espèces variable (Tableau 1). 
Tableau 1. Nombre de taxons utilisés par les 6 indices étudiés (d'après Coste et al. 1991).

Table 1. Number of taxa taken into account by the 6 indices studied (from Coste et al. 1991).

\begin{tabular}{lcccccc}
\hline $\begin{array}{l}\text { Indices } \\
\text { Nombre de } \\
\text { taxons }\end{array}$ & IPS & IDG & DES & SLA & ILM & CEC \\
\hline
\end{tabular}

Les notes obtenues sont ramenées à 20 pour faciliter les comparaisons. Les données biologiques et physicochimiques ont été centrées et réduites afin de donner un même poids aux différentes variables et étudiées en analyse en composantes principales. Les données physicochimiques ont été placées en variables actives et les indices diatomiques en variables supplémentaires. La signification des coefficients de corrélation a été testée suivant le test $r$ pour $n-2 \leqslant 100$ et suivant le test $t$ de Student pour $n-2$ $>100$. Dans ce cas, la valeur de $\mathrm{r}$ a été transformée en valeur de $t$ avec $t=r \sqrt{n-2} / \sqrt{1-r^{2}}$ (Scherrer 1984). 67 stations appartiennent au Réseau National de Bassin (RNB) et font l'objet à ce titre de mesures physiques et chimiques mensuelles. Ceci a permis d'étudier la signification des indices diatomiques de septembre par rapport aux mesures physiques et chimiques de septembre (S.) mais aussi d'août (A.), juin (J.), juillet (JL.) et aux données moyennes obtenues sur plusieurs mois (S.A., S.A.JL., S.A.JL.J., J.JL.A., J.JL.).

\section{Résultats}

\subsection{Caractérisation chimique des eaux du bassin Artois-Picardie}

Une analyse en composantes principales a été réalisée sur 174 échantillons correspondant à la campagne de septembre durant laquelle les pigments ont été mesurés. Le premier axe totalise $36,3 \%$ de la variance totale pour 14 variables chimiques. Il oppose l'ammonium $\left(\mathrm{NH}_{4}{ }^{+}\right)$, la $\mathrm{DCO}$, la $\mathrm{DBO}_{5}$ et les orthophosphates $\left(\mathrm{PO}_{4}{ }^{3}\right)$ à l'oxygène dissous $\left(\mathrm{O}_{2}\right)$ (Fig. 1) et peut être interprété comme un axe de pollution organique dégradable. L'axe 2 est déterminé par le $\mathrm{pH}$ et $\mathrm{l}^{\prime} \mathrm{O}_{2}$ et contribue pour $15 \%$ à la variance totale. Il caractérise aussi bien des stations lotiques très polluées où des concentrations en $\mathrm{NH}_{4}+$ supérieures à $100 \mathrm{mg} / \mathrm{l}$ induisent des $\mathrm{pH}$ élevés que des stations lentiques fortement eutrophisées qu'elles soient continentales ou saumâtres.
Ce sont alors des canaux littoraux (Fig. 2). Le troisième axe (11\% de la variance totale) oppose essentiellement les nitrates $\left(\mathrm{NO}_{3}{ }^{-}\right)$à la chlorophylle (Chl.) et peut être interprété comme un axe d'eutrophisation (Fig. 3).

\subsection{Signification des indices diatomiques sur le bas- sin Artois-Picardie}

525 espèces et variétés différentes ont contribué au calcul de six indices diatomiques. Les résultats montrent que ces indices donnent des réponses similaires (Tableau 2). Trois principaux facteurs sont responsables des différences observées : le nombré d'espèces prisès en compte par ces indices (Tableau 3), la nature des espèces et enfin, pour les espèces communes à ces indices, la valeur des coefficients $\mathrm{s}$ et $\mathrm{v}$.

Tableau 2. Matrice de corrélation entre 6 indices diatomiques appliqués à 355 relevés du bassin Artois-Picardie. Tous les coefficients sont significatifs à $\mathrm{p} \leqslant 0,01$.

Table 2. Correlation matrix between 6 diatom indices applied to the Artois-Picardie network. All the correlations are significant at $p \leqslant 0,01$.

\begin{tabular}{|c|c|c|c|c|c|c|}
\hline & IPS & IDG & DES & SLA & ILM & CEC \\
\hline IPS & 1 & & & & & \\
\hline IDG & 0,754 & 1 & & & & \\
\hline DES & 0,862 & 0,554 & 1 & & . & \\
\hline SLA & 0,765 & 0,414 & 0,786 & 1 & & \\
\hline ILM & 0,884 & 0,599 & 0,883 & 0,877 & 1 & \\
\hline CEC & 0,91 & 0,668 & 0,825 & 0,702 & 0,829 & 1 \\
\hline
\end{tabular}

Tableau 3. Nombre de taxons utilisés pour le calcul de chaque indice (le nombre total de taxons identifiés est de 525).

Table 3. Number of taxa used by every index for the calculation of the scores (the number of identified taxa is 525).

\begin{tabular}{lcccccc}
\hline & IPS & IDG & DES & SLA & ILM & CEC \\
Nombre de & 495 & 503 & 110 & 224 & 174 & 232 \\
taxons utilisés & 49,2 & 99,9 & 22 & 44,4 & 34,5 & 46 \\
\hline Pourcentage & 99,2 &
\end{tabular}

Ainsi, l'IPS, l'IDG et, de façon moindre, l'indice CEC prennent en compte les espèces saumâtres contrairement aux indices ILM, DES et SLA. De la même façon, seuls l'IPS, l'IDG et l'ILM tiennent compte des diatomées centriques indicatrices d'eutrophisation. L'étude de la signification de ces 6 indices sur le bassin a permis de les classer en 2 catégories (Prygiel \& Coste, 1993). L'IPS, l'IDG, 


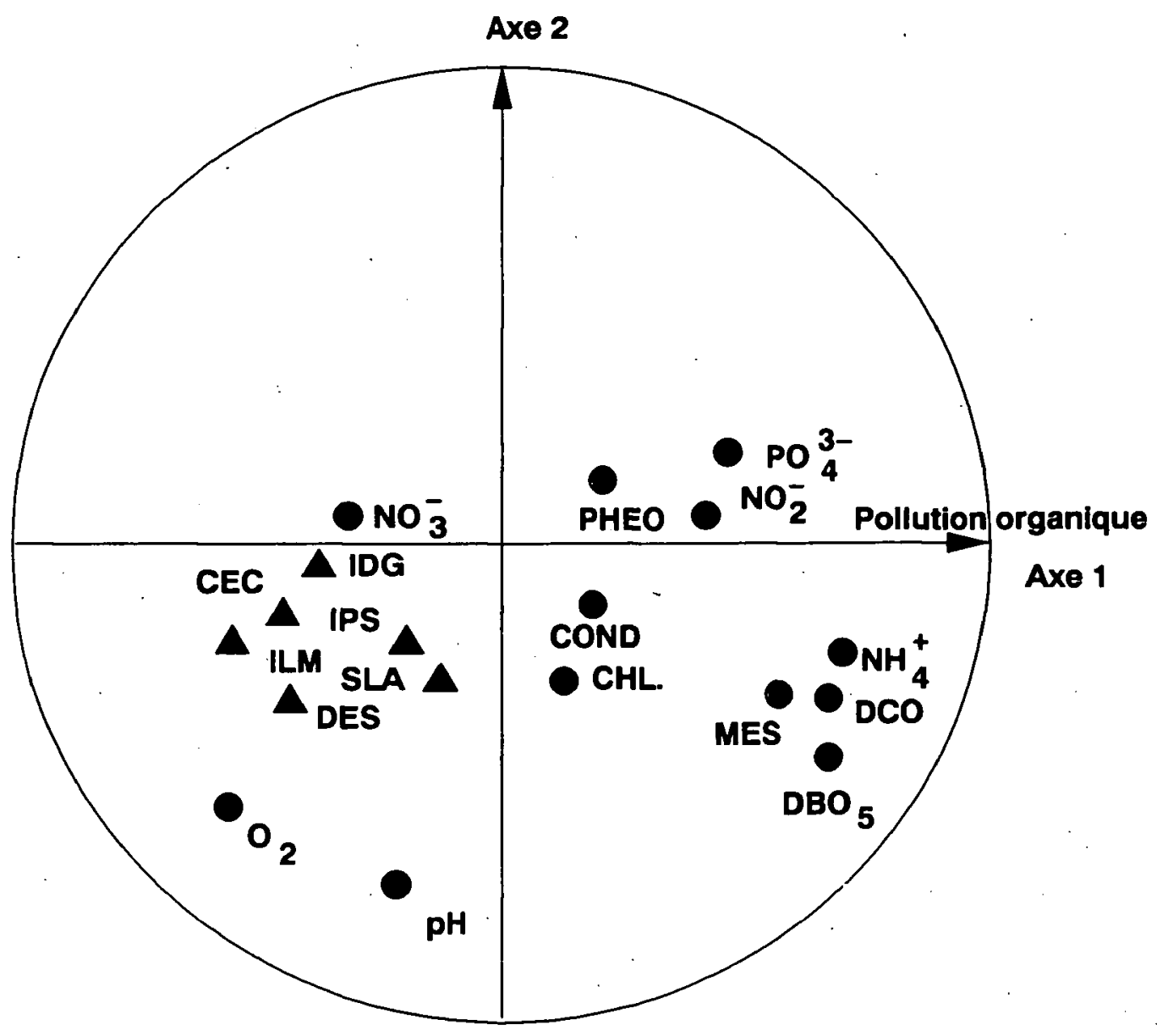

Fig. 1. Jeu de données de septembre $(n=174)$. Plan factoriel principal de l'analyse en composantes principales réalisée sur les variables chimiques. Les variables biologiques sont en variables supplémentaires. Voir le texte pour l'interprétation. CHL. = chlorophylle ; COND = conductivité ; MES = matières en suspension totales ; PHEO = phéopigments. CEC $=$ Indice de Descy \& Coste (1991) ; DES = indice de Descy (1979) ; IDG = indice diatomique générique (Coste \& Ayphassorho, 1991) ; ILM = indice de Leclercq \& Maquet (1987) ; IPS = indice de polluosensibilité spécifique (Coste in Cemagref, 1982); SLA = indice de Slàdeček (1986).

Fig. 1. September data set $(n=174)$. First plane of the principal component analysis performed on the chemical variables. Biological variables are considered as illustrative variables. See explanation in the text. CHL. = chlorophyll ; COND = conductivity ; MES $=$ total suspended matter $;$ PHEO = pheopigments. CEC = Descy \& Coste's index (1991) ; DES = Descy's index (1979) $;$ IDG $=$ Generic diatom index (Coste \& Ayphassorho, 1991) ; ILM = Leclercq \& Maquet's index (1987) ; IPS = specific polluosensitivity index (Coste in Cemagref, 1982); SLA Slàdeček's index (1986).

l'indice CEC et, dans une moindre mesure, l'ILM donnent une évaluation réaliste de la qualité générale des eaux du bassin Artois-Picardie en intégrant les phénomènes de pollution organique, de salinité et l'eutrophisation alors que les indices de Descy (1979) et de Slàdeček (1986) ne prennent en compte que la pollution organique. L'indice générique (IDG) constitue un cas particulier. Cet indice a été créé spécialement en vue d'une utilisation pratique en routine à l'échelle d'un grand bassin hydrographique. Il possède une sensibilité plus faible vis-àvis de la pollution organique (Tableau 4). Il rend cependant compte de façon satisfaisante des phénomènes de salinité exprimés par la conductivité, les chlorures et les sulfates ainsi que des phénomènes d'eutrophisation (nitrates et surtout phosphates, chlorophylle Tableau 4). Le regroupement d'espèces possédant des caractéristiques écologiques différentes entraîne, sans aucun doute, une perte de sensibilité pour la mesure de la seule pollution organique. Il est vraisemblable toutefois que la prise en compte de certains genres homogènes comme Stephanodiscus ou Entomoneis entraîne en contre partie une meilleure sensibilité pour l'estimation de mesure des phénomènes d'eutrophisation et de salinité.

L'aptitude des indices diatomiques à traduire des phénomènes tels que la pollution organique, la salinité ou l'eutrophisation découle des différentes réponses des indices aux paramètres chimiques. 


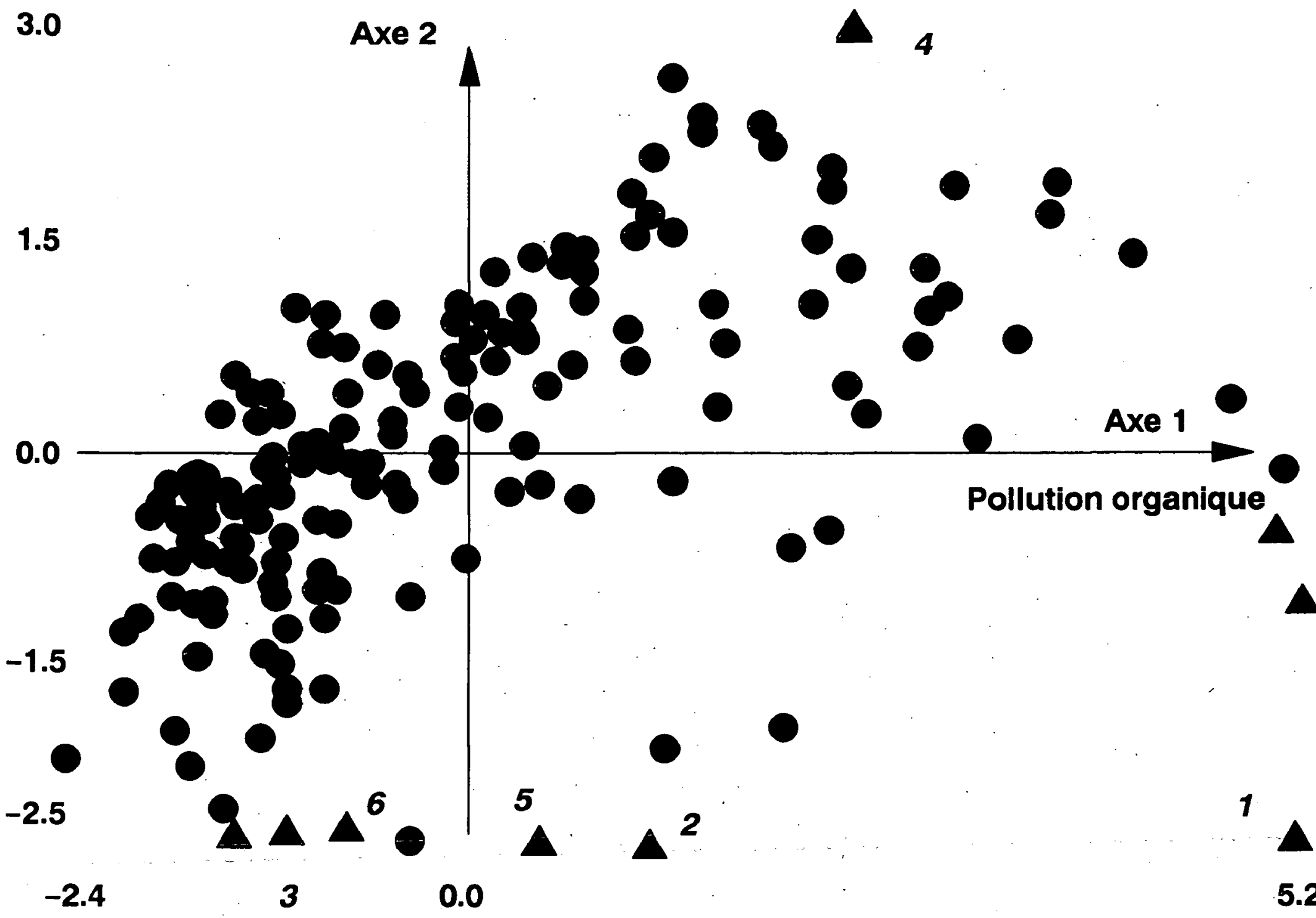

Fig. 2. Jeu de données de septembre $(n=174)$. Répartition des stations de prélèvement sur le plan factoriel principal. Les stations 1 à 6 décrivent l'axe 2 . La station 1 est une station continentale où le $\mathrm{pH}$ relativement élevé $(\mathrm{pH}=8,8)$ est dû à $\mathrm{NH}_{4}+\left(118 \mathrm{mg}^{\circ}\right)$; la concentration en $\mathrm{O}_{2}$ est nulle. La station 2 est une station littorale $\left(\mathrm{NH}_{4}{ }^{+}=18 \mathrm{mg} / 1 ; \mathrm{O}_{2}=16,2 \mathrm{mg} / 1 ; \mathrm{pH}=8,5\right.$ et conductivité $=4.000 \mu \mathrm{S} / \mathrm{s}$ ). Les stations 3 et 5 sont des stations littorales non polluées et eutrophisées (conductivité $=8.000$ et $21.000 \mu \mathrm{S} / \mathrm{s}$ et $\left.\mathrm{O}_{2}=14 \mathrm{mg} / \mathrm{l}\right)$. La station 4 est une station continentale polluée et non eutrophisée $\left(\mathrm{NH}_{4}^{+}=4,2 \mathrm{mg} / 1 \mathrm{et} \mathrm{O}_{2}=3,4 \mathrm{mg} / \mathrm{l}\right)$. La station 6 est une station continentale fortement eutrophisée $\left(\mathrm{pH}=8,9 ; \mathrm{O}_{2}=17,5 \mathrm{mg} / 1 \mathrm{et} \mathrm{NH}_{4}{ }^{+}=0 \mathrm{mg} / \mathrm{l}\right)$.

Fig. 2. September data set $(n=174)$. Distribution of the sampling stations on the first plane of the PCA. The second axis is described by the stations 1 to 6 . Station 1 is an inland site where high $\mathrm{pH}(\mathrm{pH}=8,8)$ is due to $\mathrm{NH}_{4}^{+} \cdot$; The is no $\mathrm{O}_{2}$. Station 2 is a littoral site $\left(\mathrm{NH}_{4}{ }^{+}=18 \mathrm{mg} / 1 ; \mathrm{O}_{2}=16,2 \mathrm{mg} / 1 ; \mathrm{pH}=8,5 ;\right.$ Conductivity $\left.=4.000 \mu \mathrm{S} / \mathrm{s}\right)$. Stations 3 and 5 are eutrophic littoral but unpolluted sites (conductivity $=8.000$ and $21.000 \mu \mathrm{S} / \mathrm{s}$ and $\mathrm{O}_{2}=14 \mathrm{mg} / \mathrm{l}$ ). Station 4 is a polluted inland site without eutrophication $\left(\mathrm{NH}_{4}{ }^{+}=4,2 \mathrm{mg} / \mathrm{l}\right.$; and $\left.\mathrm{O}_{2}=3,4 \mathrm{mg} / \mathrm{l}\right)$. Station 6 is a highly eutrophic inland site $\left(\mathrm{pH}=8,9 ; \mathrm{O}_{2}=17,5 \mathrm{mg} / 1\right.$ and $\left.\mathrm{NH}_{4}^{+}=0 \mathrm{mg} / \mathrm{l}\right)$.

Tous les indices présentent d'excellentes corrélations avec l'ensemble des paramètres exprimant la pollution organique. De la même façon, l'aptitude des indices IPS, IDG, CEC à traduire de façon satisfaisante la salinité est due aux bonne corrélations qu'ils présentent avec la conductivité, les chlorures et les sulfates. L'aptitude des indices IPS, IDG, ILM et CEC à exprimer les différentes formes d'eutrophisation est due aux bonnes corrélations avec les phosphates, la chlorophylle et les nitrates. 


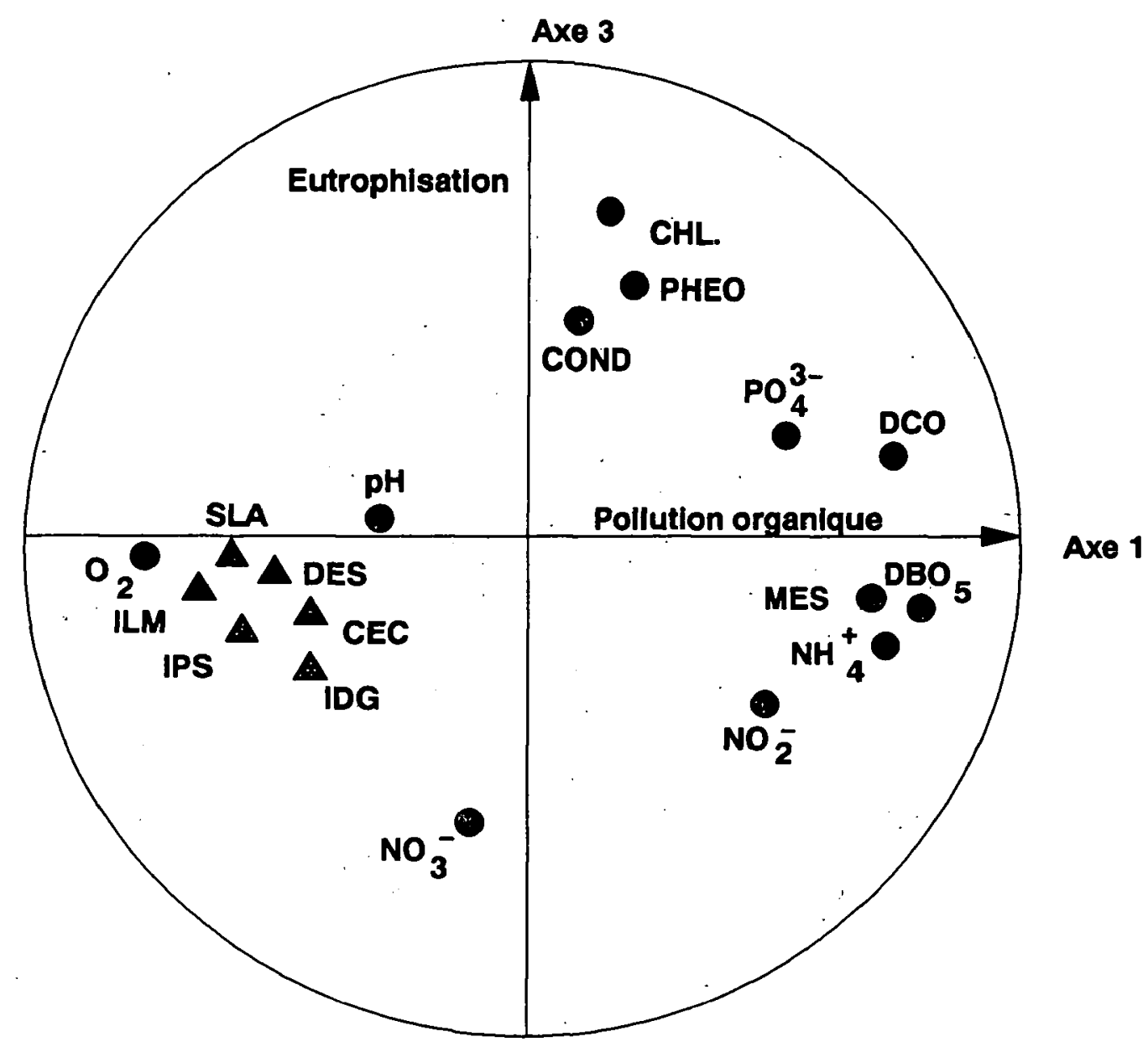

Fig. 3. Jeu de données de septembre $(n=174)$. Axes 1 et 3 de l'analyse en composantes principales réalisée sur les variables chimiques. Les variables biologiques sont en variables supplémentaires. Voir le texte pour l'interprétation. $\mathrm{CHL}$. $=$ chlorophylle $; \mathrm{COND}=$ conductivité $;$ MES = matières en suspension totales ; PHEO = phéopigments. CEC = Indice de Descy et Coste (1991); DES = indice de Descy (1979) ; IDG = indice diatomique générique (Coste \& Ayphassorho, 1991) ; ILM = indice de Leclercq et Maquet (1987) ; IPS = indice de polluosensibilité spécifique (Coste in Cemagref, 1.982); SLA = indice de Slàdeček (1986).

Fig. 3. September data set $(n=174)$. Axes 1 and 3 of the principal component analysis performed on the chemical data. Biological variables are considered as illustrative variables. See explanation in the text. CHL. = chlorophyll ; COND $=$ conductivity ; MES $=$ total suspended matter $;$ PHEO = pheopigments. CEC = Descy and Coste's index (1991) ; DES = Descy's index (1979) ; IDG $=$ Generic diatom index (Coste \& Ayphassorho, 1991) ; ILM = Leclercq and Maquet's index (1987) ; specific polluosensitivity index (Coste in Cemagref, 1982) ; SLA Slàdeček's index (1986).

La nécessité d'intégrer ces trois phénomènes pour obtenir une évaluation réaliste de la qualité des eaux a. conduit à retenir l'IPS pour l'édition de la première carte de la qualité biologique des cours d'eau du bassin Artois-Picardie. La stabilité des conditions physico-chimiques des cours d'eau du bassin ArtoisPicardie constitue un facteur favorable à l'utilisation des indices diatomiques. En effet, les diatomées ont un cycle de vie relativement court. Toutefois, les résultats montrent que les indices calculés en septembre permettent d'exprimer la qualité estivale des cours d'eau (Tableau 5).

\subsection{Ebauche de mise au point d'un outil pratique}

Bien que certaines sensibilités spécifiques et valeurs indicatrices aient été attribuées de façon parfois empirique, les résultats montrent que les indices diatomiques et plus spécialement l'IPS, permettent une estimation précise de la qualité des eaux en Artois-Picardie. L'IPS demeure toutefois difficile dans sa mise en œuvre car il requiert une identification à l'espèce de l'ensemble des espèces rencontrées. Aussi différentes approches ont-elles été tentées en vue de l'établissement d'un indice pratique et utilisable en routine sur un grand bassin hydrographique.

\subsubsection{Approche générique}

La corrélation IPS-IDG reste relativement faible (Tableau 2, Fig. 4) et ce, malgré l'introduction de nouveaux genres récemment décrits (Round et al. 1990) en vue d'affiner le diagnostic. 
Tableau 4. Corrélations entre les principaux paramètres responsables des variations de la qualité des eaux et les indices diatomiques. $\mathrm{NH} 4=$ ammonium ; PO4 = orthophosphates ; DBO5 et DCO = demandes biochimique et chimique en oxygène dissous ; O2 $=$. oxygène dissous $; \mathrm{COND}=$ conductivité $; \mathrm{NO} 3=$ nitrates. $\mathrm{Chl}$. = chlorophylle ; $\mathrm{CL}=$ chlorures ; SO4 = sulfates. Les corrélations ont été établies sur le jeu de données de septembre $(n=174)$ sauf pour CL et $\mathrm{SO} 4(\mathrm{n}=114)$. Seuils de signification : ${ }^{* * *}: \mathrm{p} \leqslant 0,001$; ${ }^{* *}: \mathrm{p} \leqslant 0,001 ;{ }^{*}: \mathrm{p} \leqslant 0,05$.

Table 4. Correlations between the main parameters responsible for water quality variations and the diatom indices. NH4 = ammonia ; PO4 = phosphates $; \mathrm{BD} 05$ and $\mathrm{DCO}=$ biochemical and chemical oxygen demands $; \mathrm{O} 2=$ dissolved oxygen $; \mathrm{COND}=$ conductivity $; \mathrm{NO3}=$ nitrates $; \mathrm{Chl} .=$ chlorophyll $; \mathrm{CL}=$ chlorides $; \mathrm{S}=$ sulfates. The correlations have been calculated on the September data set $(n=114)$ except $C L$ and SO4 $(n=114)$. Level of significance $:^{* * *}: p \leqslant 0,001 ;{ }^{* *}: p \leqslant 0,01 ;{ }^{*}: p \leqslant 0,05$.

\begin{tabular}{|c|c|c|c|c|c|c|}
\hline Paramètre & IPS & IDG & DES & SLA & ILM & CEC \\
\hline $\mathrm{NH} 4$ & $0,38^{\star \star \star}$ & $0,25^{\star \star \star}$ & 0,38 \# & $0,39 \star \star \star$ & $0,43^{\star \star \star}$ & $0,33^{\star \star}$ \\
\hline PO4 & $0,55^{\star \star \star}$ & $0,4^{\star \star *}$ & $0,5^{\star \star \star}$ & $0,4^{\star \star \star}$ & $0,54^{\star \star \star}$ & $0,46 \star \star$ \\
\hline DBO5 & $0,25^{\star \star x}$ & $0,18^{\star}$ & $0,19^{\star \star}$ & $0,22^{\star \star}$ & $0,27^{\star \star \star}$ & $0,23^{\star \star}$ \\
\hline DCO & $0,39 \star \star$ & $0,36 \star \star \star x$ & $0,23^{\star \star}$ & 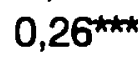 & $0,38 * \star \star$ & 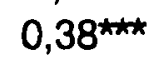 \\
\hline $\mathrm{O} 2$ & $0,49^{\star \star \star}$ & $0,33^{\star \star \star}$ & $0,56 \ldots$ & $0,46 \star \star$ & $0,55^{\star \star \star}$ & $0,45^{\star \star \star}$ \\
\hline $\mathrm{pH}$ & 0,15 & 0,01 & $0,29 \star \star$ & $0,18 \star$ & $0,22^{\star \star}$ & 0,13 \\
\hline COND & $0,17^{\star}$ & $0,23^{\star \star}$ & 0,01 & 0,05 & 0,1 & 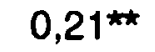 \\
\hline NO3 & $0,22^{\star \star}$ & $0,22^{\star}$ & 0,09 & 0,1 & $0,19^{\star}$ & $0,19^{\star}$ \\
\hline Chl. & $0,2^{\star \star}$ & $0,23^{\star \star}$ & 0,06 & 0,09 & $0,17^{\star}$ & $0,22^{\star \star}$ \\
\hline $\mathrm{CL}$ & 0,16 & $0,24^{\star}$ & 0,01 & 0,17 & 0,1 & $0,23^{*}$ \\
\hline SO4 & $0,23^{*}$ & $0,24^{\star}$ & 0,1 & 008 & 018 & $0,29^{\star \star}$ \\
\hline
\end{tabular}

Tableau 5. Corrélations entre les indices diatomiques et la pollution organique (axe 1 de l'ACP) pour différentes périodes $(n=65)$. S. = septembre $;$ A. = août ; JL. = juillet ; J. = juin. Pour les autres périodes, les corrélations ont été établies sur la base de moyennes des variables chimiques.

Table 5. Correlations between diatom indices and organic pollution (First component of the PCA) for different periods ( $\mathrm{n}=65$ ). S. $=$ September $;$ A. = August $;$ JL. = July $;$ J. = June. For other periods, the correlations have been calculated on the basis of average values of the chemical variables.

\begin{tabular}{ccccccc}
\hline & IPS & IDG & DES & SLA & ILM & CEC \\
\hline S. & $0,69^{\star \star}$ & $0,42^{\star \star}$ & $0,66^{\star \star}$ & $0,58^{\star \star}$ & $0,65^{\star \star}$ & $0,65^{\star \star}$ \\
A. & $0,66^{\star \star}$ & $0,41^{\star \star}$ & $0,63^{\star \star}$ & $0,57^{\star \star}$ & $0,61^{\star \star}$ & $0,65^{\star \star}$ \\
JL. & $0,37^{\star \star}$ & 0,12 & $0,38^{\star \star}$ & $0,27^{\star}$ & $0,31^{\star}$ & $0,31^{\star}$ \\
J. & $0,67^{\star \star}$ & $0,41^{\star \star}$ & $0,67^{\star \star}$ & $0,55^{\star \star}$ & $0,62^{\star \star}$ & $0,68^{\star \star}$ \\
J.JL.A. & $0,67^{\star \star}$ & $0,36^{\star \star}$ & $0,68^{\star \star}$ & $0,56^{\star \star}$ & $0,61^{\star \star}$ & $0,64^{\star \star}$ \\
J.JL. & $0,61^{\star \star}$ & $0,30^{\star}$ & $0,63^{\star \star}$ & $0,50^{\star \star}$ & $0,55^{\star \star}$ & $0,57^{\star \star}$ \\
A.S & $0,68^{\star \star}$ & $0,52^{\star \star}$ & $0,65^{\star \star}$ & $0,58^{\star \star}$ & $0,63^{\star \star}$ & $0,66^{\star \star}$ \\
S.A.JL. & $0,67^{\star \star}$ & $0,38^{\star \star}$ & $0,66^{\star \star}$ & $0,56^{\star \star}$ & $0,62^{\star \star}$ & $0,64^{\star \star}$ \\
S.A.JL.J. & $0,69^{\star \star}$ & $0,39^{\star \star}$ & $0,68^{\star \star}$ & $0,56^{\star \star}$ & $0,62^{\star \star}$ & $0,64^{\star \star}$ \\
\hline
\end{tabular}

IPS

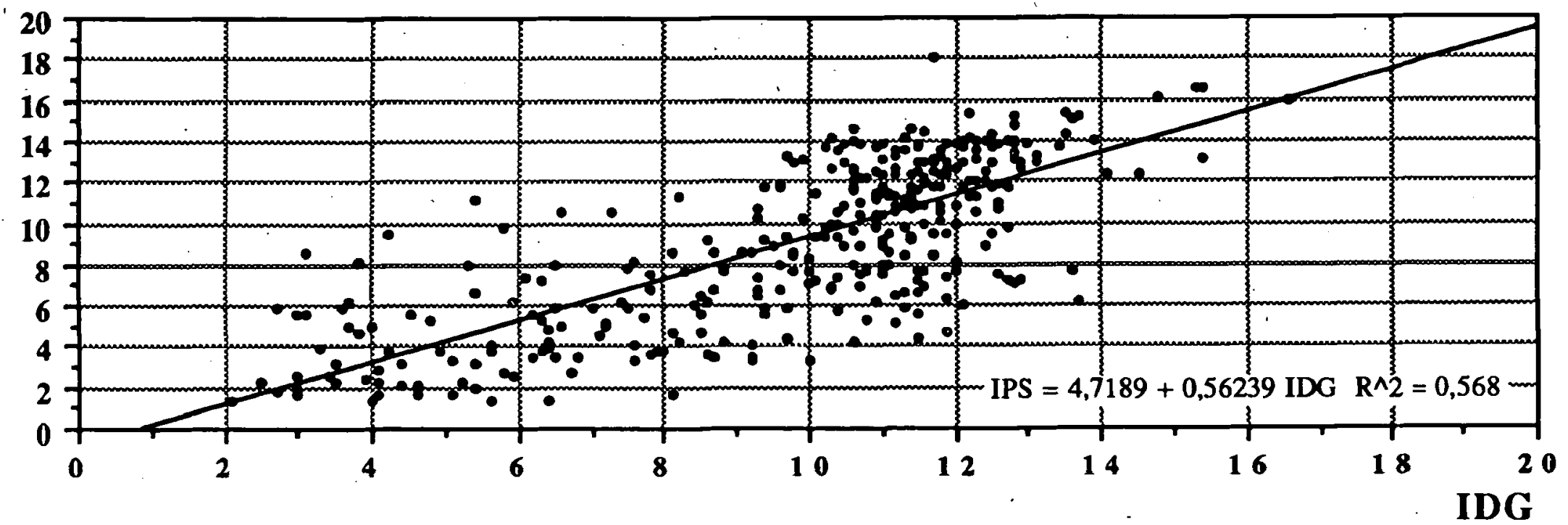

Fig. 4. Corrélation linéaire entre l'IPS et l'IDG pour 355 relevés effectués sur le bassin Artois-Picardie.

Fig. 4. Linear correlation beween SPI and GDI for 355 samples taken in the Artois-Picardie network. 
Il faut toutefois noter que la relation est sensiblement meilleure qu'avec l'IDG de Coste (in Cemagref 1982) et de Rumeau \& Coste (1988) où la classification utilisée était celle de Bourrelly (1981). L'explication réside dans l'impossibilité de classer certaines espèces autrement qu'à l'intérieur de genres peu déterminants ou très ubiquistes du point de vue de la qualité de l'eau. Lorsque ces espèces deviennent dominantes, l'écart entre les valeurs indicielles spécifiques et génériques de sensibilité à la pollution est tel qu'il entraîne un écart important sur la note globale. Ces écarts entre IPS et IDG peuvent atteindre 7,5 mais, d'une manière générale, l'IDG semble donner des valeurs plus optimistes que l'IPS sur l'ensemble des inventaires. Il faut noter que les variations des 2 indices suivent un profil sensiblement similaire (Fig. 5). 46 inventaires sur un total de 355 , montrent des écarts entre IPS et IDG supérieurs ou égaux à 4.39 traduisent une surestimation de l'IDG et 5 seulement une sous-estimation. L'examen détaillé des inventaires, permet d'identifier les espèces responsables (Tableau 6). Celles-ci sont souvent facilement identifiables. Certaines espèces ont une valeur de sensibilité à la pollution supérieure à celle du genre auquel elles appartiennent. Elles conduisent à un IPS supérieur à l'IDG et donc à une sous-estimation de l'IDG. C'est par exemple le cas de la station 22.000 , en septembre 1990 , où Achnantes minutissima et Nitzschia sublinearis représentent respectivement $48 \%$ et $25 \%$ de l'échantillon. On note alors un IPS de 18,1 et un
IDG de 11,7. A l'inverse, certaines espèces ont une sensibilité à la pollution inférieure à celle du genre auquel elles appartiennent. Elles conduisent donc à une surestimation de l'IDG. C'est le cas de la station 18.000, en septembre 1990, où Gomphonema parvulum et Achnanthes hungarica représentent respectivement $49 \%$ et $20 \%$ de l'échantillon: On obtient alors un IPS de 6,2 pour un IDG de 13,7 . Il apparaît donc indispensable, malgré la scission des genres les mieux représentés comme Nitzschia et Navicula en nombreux sous genres ou sections, de prendre en compte certaines espèces. C'est une pratique similaire qui a fait le succès des indices biotiques fondés sur des invertébrés.

\subsubsection{Approche spécifique}

Des simulations ont été effectuées à partir d'inventaires avec l'aide du logiciel OMNIDIA. Chaque relevé a fait l'objet de plusieurs calculs indiciels. Ainsi, ont été éliminées, dans un premier temps, les espèces dont l'abondance relative était inférieure à $0,5 \%$, puis les espèces dont l'abondance relative était inférieure à $1 \%, 2 \%, 5 \%$ et $10 \%$. Les résultats montrent que si l'on accepte des variations de l'ordre de 0,5 , la valeur de l'indice est, en règle générale, déterminée par les espèces dont l'abondance relative est supérieure à $2 \%$. Il existe toutefois des exceptions. Ainsi, sur la Haute Meldyck à Saint-Omer (IPS $=1,8)$, seules deux espèces sur $30:$ Nitzschia palea $(\mathrm{A}=63 \%)$ et Cyclotella meneghiniana $(\mathrm{A}$ $=10 \%$ ), sont responsable de la valeur indicielle.

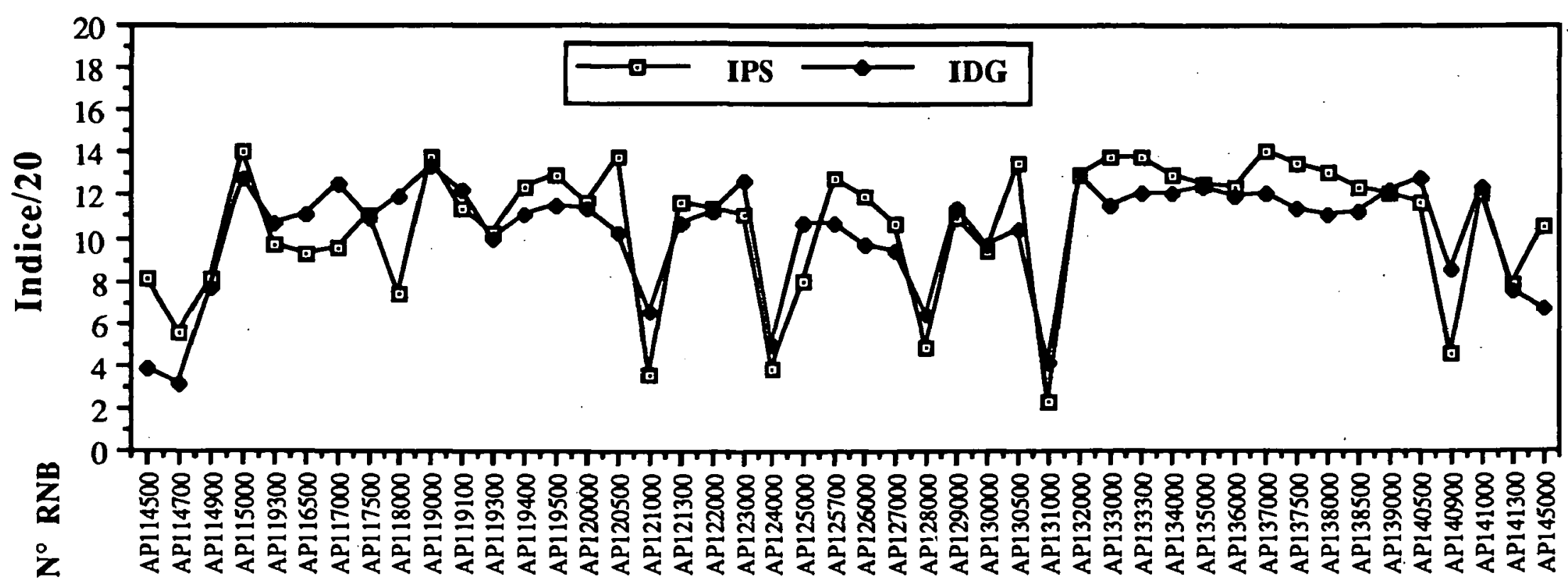

Fig. 5. Ecarts entre les valeurs de l'IPS et de l'IDG pour quelques stations du réseau de mesure du bassin Artois-Picardie.

Fig. 5. Differences between SPI and GDI score values for some stations belonging to the Artois-Picardie network. 
Tableau 6. Valeurs des coefficients de sensibilité à la pollution (s) et de valeur indicatrice (v) de quelques espèces et des genres correspondants pour les indices IPS (Coste in Cemagref 1982) et IDG (Coste \& Ayphassorho 1991). * : espèces à l'origine d'écarts entre IPS et IDG supérieurs ou égaux à 4.

Table 6. Values of the coefficients of sensitivity (s) and indicator value (v) of some species and their corresponding genera for IPS (Coste in Cemagref 1982) and IDG (Coste \& Ayphassorho 1991). * : species responsible for differences greater than 4 between IPS and IDG.

\begin{tabular}{|c|c|c|c|c|c|}
\hline Genre & $\mathbf{s}$ & $\mathbf{v}$ & Espèce & $\mathbf{s}$ & $\mathbf{v}$ \\
\hline \multirow[t]{4}{*}{ Achnanthes } & 4,5 & 2,1 & A. hungarica Grünow * & 2 & 3 \\
\hline & & & A. lanceolata (Breb.) spp. frequentissima Lange-Bertalot & 2,8 & 1 \\
\hline & & & A. minutissima fo. minutissima Kützing * & 5 & 1 \\
\hline & & & A. brevipes var. intermedia (Kütz.) Cleve & 3 & 2 \\
\hline \multirow[t]{2}{*}{ Amphora } & 2,6 & 2,2 & A. pediculus Kützing * & 4 & 1 \\
\hline & & & A. libyca Ehr. & 4 & 2 \\
\hline \multirow[t]{2}{*}{ Aulacosira } & 3,8 & 1,4 & A. granulata (Ehr.) Simonsen * & 2,9 & 1 \\
\hline & & & A. ambigua (Grün.) Simonsen & 3 & 1 \\
\hline \multirow[t]{2}{*}{ Cyclotella } & 3,7 & 1,7 & C. atomus Hustedt * & 2 & 1 \\
\hline & & & C. meneghiniana Kützing * & 2 & 1 \\
\hline Cocconeis & 3,5 & 1,8 & C. placentula Ehr. var. euglypta (Ehr.) Grünow * & 3 & 1 \\
\hline Craticula & 2,2 & 3 & C. accomoda (Hustedt) D.G. Mann * & 1 & 3 \\
\hline Fragilaria & 3,6 & 1,7 & F. ulna (Nitzsh.) Lange-Bertalot * & 3 & 1 \\
\hline \multirow[t]{2}{*}{ Gomphonema } & 3,6 & 1,9 & G. parvulum Kützing * & 2 & 1 \\
\hline & & & G. grovei var. lingulatum (Hust.) Lange-Bertalot & 2 & 3 \\
\hline \multirow[t]{4}{*}{ Melosira } & 3,5 & 1,7 & M. nummuloides (Dillwyn) Agardh * & 2 & 3 \\
\hline & & & M. lineata (Dillwyn) Agardh & 2 & 3 \\
\hline & & & M. undulata (Ehr.) Kützing & 2 & 3 \\
\hline & & & M. varians Agardh * & 4 & 1 \\
\hline \multirow[t]{7}{*}{ Navicula } & 3,4 & 1,9 & N. lanceolata Agardh (Ehr.) * & 3 & 1 \\
\hline & & & N. minima Grünow * & 3 & 1 \\
\hline & & & N. saprophila Lange-Bertalot \& Bonik * & 2 & 1 \\
\hline & & & N. subminuscula Manguin * & 2 & 1 \\
\hline & & & N. subrotundata Hustedt * & 2,3 & 1 \\
\hline & & & N. tripunctata (O.F.M.) Bory * & 4 & 2 \\
\hline & & & N. veneta Kützing * & 1 & 2 \\
\hline \multirow[t]{8}{*}{ Nitzschia } & 1 & 2,3 & N. amphibia Grünow * & 2 & 2 \\
\hline & & & N. capitellata Hustedt * & 1 & 3 \\
\hline & & & N. fonticola Grünow * & 3 & 1 \\
\hline & & & N. frustulum Kützing * & 2 & 1 \\
\hline & & & N. linearis (Ag.) W. Smith & 3 & 2 \\
\hline & & & N. palea (Kützing) W. Smith * & 1 & 3 \\
\hline & & & N. subacicularis Hustedt * & 3 & 3 \\
\hline & & & N. sublinearis Hustedt * & 5 & 2 \\
\hline \multirow[t]{2}{*}{ Sellaphora } & 2,8 & 1,7 & S. pupula (Kützing) Mereschkowski D. G. Mann * & 2 & 2 \\
\hline & & & S. seminulum (Grünow) D.G. Mann * & 1 & \\
\hline Stephanodiscus & 2,9 & 1,4 & S. hantzschii fo. tenuis (Hustedt) Hakansson \& Stoermer * & 3 & \\
\hline
\end{tabular}


A l'inverse, sur le Doulàc à Saint-Omer (IPS = 2.9), il faut retenir toutes les espèces dont l'abondance relative est supérieure à $0,5 \%$ soit 17 espèces sur 56 répertoriées. Dans tous les cas, les espèces qui n'apparaissent qu'en un ou deux exemplaires ne contribuent qu'au bruit de fond. Finalement, le facteur déterminant est la structure de l'échantillon. Lorsque quelques espèces sont responsables de la quasi-totalité des effectifs, on peut ne pas tenir compte des autres. Par contre, lorsque la diversité selon Shannon \& Wiener (in Legendre \& Legendre 1984) augmente, il devient indispensable de considérer les espèces présentes en quelques exemplaires. Il apparaît donc très difficile de généraliser une telle pratique. On peut toutefois remarquer que pour des opérations de pure routine dont le but est une évaluation générale de la qualité de l'eau, une telle pratique peut se justifier. En effet, les espèces les plus abondantes conduisent certes à des écarts quelquefois importants mais qui ne remettent généralement pas en cause la classe de qualité déterminée à l'aide de l'ensemble des espèces inventoriées.

\subsubsection{Réduction des effectifs à compter}

Que ce soit dans le but de décrire les peuplements diatomiques ou de calculer des indices, les comptages portent sur plusieurs centaines d'individus par préparation, de 250 (Sabater et al: 1987) à 400 (Lafont et al. 1988, Prygiel 1991) voire 500 (Coste in Cemagref 1982, Descy 1979, Descy \& Coste 1991, Leclercq 1988). Afin d'apprécier le degré de fiabilité des estimations indicielles susceptible de varier en fonction du nombre d'individus comptés, des comptages fractionnés (tous les 20 individus) ont été réalisés et les indices correspondants calculés (Coste \& Ayphassorho 1991). Les résultats de ces comptages fractionnés montrent des situations variables. Dans certains cas, un dénombrement portant sur une centaine d'individus semble suffisant pour l'obtention d'un indice stabilisé (Fig. 6). Dans d'autres cas (Fig. 7), il semble nécessaire de poursuivre les comptages jusqu'à des effectifs de 300 à 350. Dans tous les cas, 400 individus au moins, sont nécessaires pour préserver la représentativité du nombre d'espèces et permettre des investigations ultérieures à caractère plys typologique.

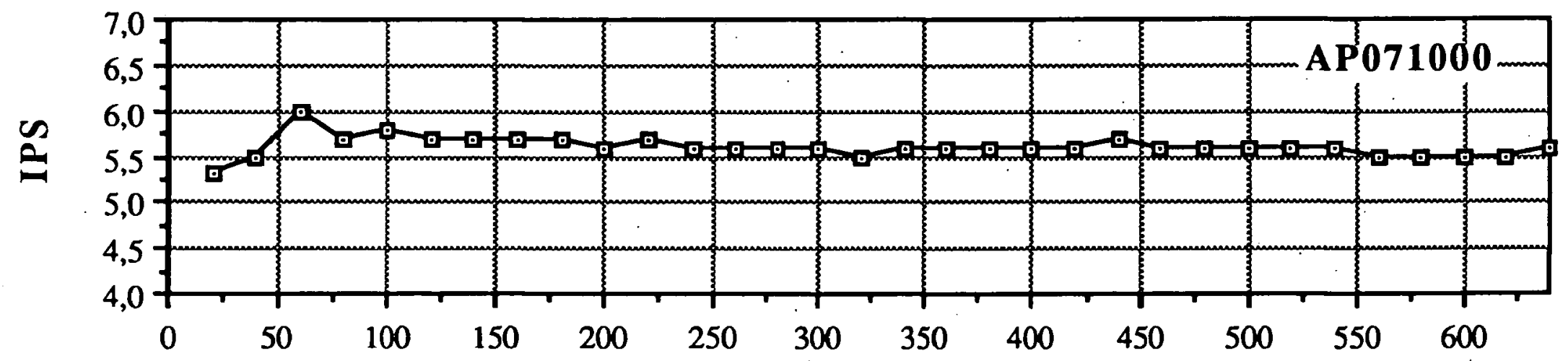

Fig. 6. Evolution de l'IPS en fonction de l'effectif compté pour la station 71.000 du réseau Artois-Picardie. Une centaine d'individus suffit pour l'obtention d'un indice stable.

Fig. 6. Evolution of SPI according to the number of cells counted for the station 71.000 of the Artois-Picardie network. 100 cells are enough to obtain a stable index.

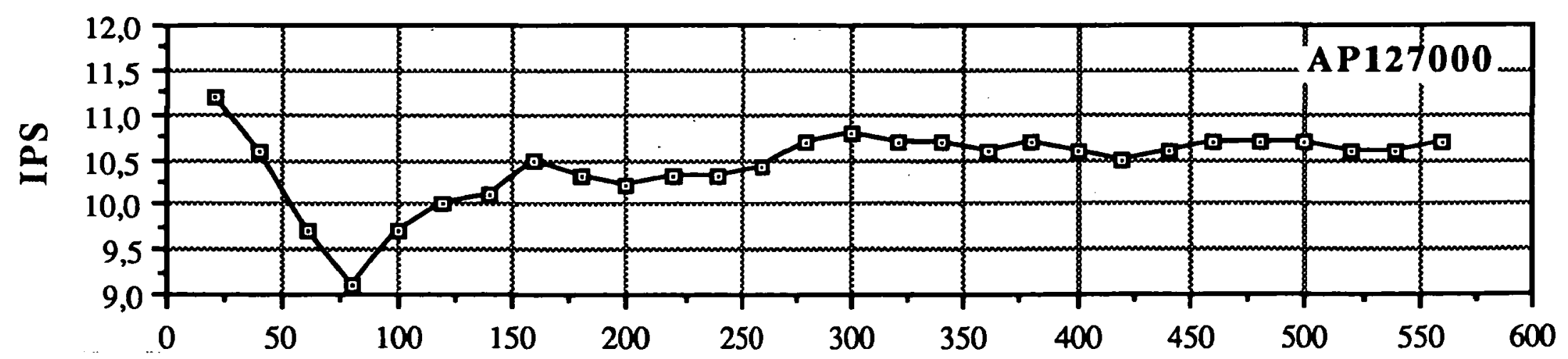

Fig. 7. Evolution de l'IPS en fonction de l'effectif compté pour la station 127.000 du réseau Artois-Picardie. 300 individus sont nécéssaires pour l'obtention d'un indice stable.

Fig. 7. Evolution of SPI according to the nimber of cells counted for the station 127.000 of the Artois-Picardie network. 300 cells are enough to obtain a stable index. 


\section{Discussion}

Bien que considérées comme faisant partie des meilleurs bioindicateurs pour l'évaluation de la qualité des eaux continentales courantes, les diatomées demeurent relativement peu utilisées (Descy 1973, Descy.1976, Leclercq \& Vandevenne 1987, Sabater et al. 1987, 1988, Vanlandingham 1976). L'Indice Diatomique Générique proposé par Coste (in Cemagref 1982) et repris par Rumeau \& Coste (1988) marquait une étape importante en proposant aux organismes gestionnaires de la qualité des eaux, un outil pratique dans sa conception et sa mise en œuvre. Les récents travaux menés au sein de grands bassins hydrographiques (A.E. Artois-Picardie 1991, A.E. Rhône-Méditerranée-Corse 1989) et ceux actuellement en cours sur le bassin Rhin-Meuse, montrent bien l'intérêt de ces organismes pour ce type d'approche. Cet intérêt est d'autant plus grand que les algues apportent des informations complémentaires à celles fournies par les macroinvertébrés (Lafont et al. 1988), en particulier dans la mise en évidence de phénomènes fugaces. Cependant, lorsque les conditions chimiques de l'eau sont stables, les diatomées et les indices diatomiques peuvent intégrer la qualité générale de l'eau sur des périodes relativement longues comme cela a été montré par Leclercq (1989) et Prygiel \& Coste (1993). Parmi les différentes options susceptibles de conduire à la mise au point d'un indice pratique, il semble que l'approche générique, avec, dans certains cas, des déterminations spécifiques constitue une des approches les plus satisfaisantes pour les gestionnaires. D'une part, cette approche utilise l'ensemble des taxons rencontrés au cours de l'inventaire.D'autre part, il semble possible de regrouper des espèces au sein de genres ou même de sections possédant une même sensibilité à la pollution. Les identifications spécifiques ne portent alors que sur quelques espèces dont la sensibilité à la pollution est très différente de celle du genre auquel elles appartiennent. Le caractère halophile ou saprophile est le plus souvent l'élément le plus discriminant de ces variations. Les particularités thermiques ou la polluo-sensibilité globale d'une espèce peuvent également l'éloigner des caractéristiques moyennes du genre. Le genre Achnantes regroupe en majeure partie des espèces d'eaux courantes fraîches et oxygénées avec une sensibilité souvent marquée aux pollutions $(\mathrm{s}=4,5)$. Par contre, Achnantes hungarica $(\mathrm{s}=2$ ) est une espèce d'eaux calmes supportant des teneurs en sels ammoniacaux élevées et est souvent épiphyte sur les lentilles d'eau, elles-mêmes utilisées dans les processus d'épuration extensifs (lagunage). $A$. brevipes $(\mathrm{s}=3), A$. subsalsa $(\mathrm{s}=2,5)$ sont des formes halophiles et $A$. thermalis $(\mathrm{s}=2)$ peut supporter des températures supérieures à $40^{\circ} \mathrm{C}$.

Les Amphora sont scindés en deux groupes : les formes halophiles et les formes d'eau douce moins nombreuses et"plus sensibles ( $A$. pediculus, $\mathrm{s}=4$ ).

Le genre Aulacoseira comporte des formes lacustres de milieu oligo ou mésotrophe et des formes eutrophes souvent abondantes dans le potamoplancton comme $A$. granulata (s $=2,9$ ).

Chez le genre Cyclotella, la majorité des espèces affectionne les eaux calmes où elles sont souvent considérées comme formes pionnières (ex. Cyclotella ocellata dans les ballastières). $C$. meneghiniana et C. atomus $(\mathrm{s}=2)$, espèces saprophiles susceptibles de nutrition hétérotrophe (Cholnoky 1968), échappent à la règle et font chuter sensiblement, avec quelques espèces halophiles, la sensibilité globale du genre.

Les genres Fragilaria, Gomphonema présentent peu d'espèces halophiles ou saprophiles et les espèces citées dans le tableau $6(F$. ulna, $\mathrm{s}=3 ; G$. parvulum, $s=2$ ) sont parmi les plus fréquemment responsables des écarts IPS-IDG.

Le genre Melosira n'est plus représenté que par $M$. varians dans les eaux douces; en revanche, il comprend de nombreuses espèces marines ou saumâtres.

Le genre Nitzschia, retenu comme référence basse pour les valeurs de sensibilité à la pollution $(s=1)$, renferme des espèces électives de bonnes qualités d'eau ou polluo-sensibles, comme $N$. sinuata (s = 4), $N$. sublinearis $(\mathrm{s}=5), N$. acidoclinata $(\mathrm{s}=5)$, $N$. hantzschiana $(\mathrm{s}=5)$, etc... Il doit être utilisé avec précaution d'autant qu'à l'intérieur des sous-genres (section des lanceolatae), des confusions taxonomiques sont possibles entre de nombreuses espèces $(N$. frustulum, $N$. fonticola, $N$. acidoclinata).

Chez Navicula, la réhabilitation de nouveaux genres (Round et al.1990) appliquée aux diatomées du bassin Artois-Picardie, n'a pas apporté les résultats escomptés, certains remaniements taxonomiques s'avérant peu judicieux. Le genre Sellaphora (Mereschkowski) dans lequel Mann (1989) regroupe, par exemple, des espèces de la section (sous-genre) 
des Bacillares et des Entoleiae (in Hustedt 1930) ou des Minusculeae (section f, in Krammer \& LangeBertalot 1986) sur la base de critères morphologiques peu usités (structure du plaste, des aréoles et du raphé), reste très hétérogène. Placer Navicula (Sellaphora) seminulum à côté de $S$. pupula est difficilement acceptable et cette espèce devrait logiquement rester dans les Minusculeae à côté de $N$. minima, et $N$. joubaudii.

L'utilisation de nouvelles sections redéfinies par Krammer \& Lange-Bartalot dans la prochaine édition de la Sußwasserflora, devrait contribuer à une meilleure approche générique car elle est exclusivement fondée sur des caractéristiques morphologiques accessibles en microscopie photonique.

\section{Conclusion}

Les méthodes biologiques qui utilisent les diatomées s'avèrent particulièrement intéressantes pour la mesure de la qualité générale des eaux mais aussi pour l'appréciation des pollutions organique, saline ou trophique. Ce point est particulièrement important dans le bassin Artois-Picardie du fait de l'existence d'un réseau canalisé particulièrement dense et souvent fortement eutrophisé, et des problèmes de pollution saline existant sur le littoral mais aussi en milieu continental comme, par exemple, dans l'ancien bassin minier. La nécessité de suivre un vaste réseau de façon régulière implique la mise au point de techniques pratiques et rapides. L'indice diatomique générique constitue un premier pas dans cette direction. Cet indice est cependant perfectible. La meilleure solution consistera à utiliser simultanément des genres et des espèces, chaque fois que leur identification est aisée. Il faut toutefois rester conscient des limites de ce type d'approche. Il est en effet vain d'attendre de genres de diatomées, qui renferment une multitude d'espèces à spectre écologique souvent étendu, une précision suffisante pour exprimer autre chose qu'un niveau de qualité général. L'information obtenue au niveau spécifique sera toujours plus précise, en particulier dans la description de pollutions diffuses.

\section{Travaux cités}

AE Artois-Picardie. 1992. - Carte de qualité hydrobiologique des cours d'eau du bassin Artois-Picardie. Doc. A.E. ArtoisPicardie, $15 \mathrm{p}+$ carte.

Blandin P. 1986. - Bioindicateurs et diagnostic des systèmes écologiques. Bull. Ecol. 17 (4) : 215-307.
Bourrelly P. 1981. - Les algues d'eau douce. Initiation à la systématique. Tome II : les algues jaunes et brunes. Boubée \& Cie (ed.), Paris : 518 p.

Cazaubon A. 1991. - Problems and objectives of sampling river algae for monitoring. In Whitton B.A., Rott E. \& Friedrich G. (eds.), Use of algae for monitoring rivers, Düsseldorf, E. Rott, Innsbruck : 17-24.

Cemagref. 1982. - Etude des méthodes biologiques quantitatives d'appréciation de la qualité des eaux. Rapport Q.E. Lyon - A.F.B. Rhône-Méditerranée-Corse : 218 p.

Chandler J.R. 1970. - A biological approach to water quality management. Journal of water pollution control, $69 ; 415-422$.

Cholnoky B.J. 1968. - Die Ökologie der Diatomeen in Binnengewässern. Cramer Ed. : 699 p.

Coste M. 1976. - Contribution à l'écologie des diatomées benthiques et périphytiques de la Seine : distribution longitudinale et influence des pollutions. Soc. Hydrotech. Fr., 14/9 : 1-6.

Coste M. 1978. - Sur l'utilisation des diatomées benthiques pour l'appréciation de la qualité biologique des eaux courantes. Thèse Biol. vég., Univ. Besançon : $150 \mathrm{p}$.

Coste M. \& Ayphassorho (H. 1991. - Etude de la qualité des eaux du Bassin Artois-Picardie à l'aide des communautés de diatomées benthiques (Application des indices diatomiques). Rapport Cemagref Bordeaux-AE Artois-Picardie : 227 p.

Coste M., Bosca C. \& Dauta A. 1991. - Use of algae for monitoring rivers in France. In Whitton B.A., Rott E. \& Friedrich G. (eds.), Use of algae for monitoring rivers, Düsseldorf, E. Rott, Innsbruck : 75-88.

Cox E.J. 1991. - What is the basis for using diatoms as monitors of river quality. In Whitton B.A., Rott E. \& Friedrich G. (eds.), Use of algae for monitoring rivers, Düsseldorf, E. Rott, Innsbruck ; 33-40.

De Pauw N. \& Vanhooren G. 1983. - Method for biological quality assessment in Belgium. Hydrobiologia, 100 : 153-168.

Descy J.P. 1973. - La végétation algale benthique de la Meuse belge et ses relations avec la pollution des eaux. Lejeunia Nouv. ser. 66 ; 1-62.

Descy J.P. 1976. - La végétation algale benthique de la Somme (France) et ses relations avec la qualité des eaux. Mem. Soc. roy. Bot. Bel. 7 : 101-128.

Descy J.P. 1979. - A new approach to water quality estimation using diatoms. Nova Hedwigia 64 : 305-323.

Descy J.P. \& Coste M. 1989. - Application d'un nouvel indice diatomique (indice CEE 88) au réseau national de bassin Rhône-Méditerranée-Corse, rapport d'étude, Agence de l'Eau RMC : 86 p.

Descy J.P. \& Coste M. 1991. - A test of methods for assessing water quality based on diatoms. Verh. Internat. Verein. Limnol. 24 : 2112-2116.

Eloranta P. 1991. - Use of algae for monitoring rivers in Finland. In Whitton B.A., Rott E. \& Friedrich G. (eds.), Use of algae for monitoring rivers, Düsseldorf, E. Rott, Innsbruck : 71-75.

Fabri R. 1987. - Bio-indicateurs et signification des indices diatomiques de qualité des eaux. Cah. Biol. Mar. 28 : 285-289.

Fabri R. \& Leclercq L .1986. - Diatom communities in the rivers of Ardennes (Belgium) : Natural types and impact of pollutions. In M. Ricard (ed.) Proc. of the 8th Internat. diatom Symp. Paris 1984, O. Koeltz publ., Koenigstein : 337-346. 
Friedrich G. 1991. - Use of phytoplankton in monitoring rivers in Federal Republic of Germany. In Whitton B.A., Rott E. \& Friedrich G. (eds.), Use of algae for monitoring rivers, Düsseldorf, E. Rott, Innsbruck : 97-103.

Hustedt F. 1930: - Die Kieselalgen Deutschlands, Österreich und der Schweitz. In Kryptogamenflora (L. Rabenhorst) Akad. Verlag. Leipzig, $7.1: 1-920$.

Krammer K. \& Lange-Bertalot (H.). 1986. - Bacillariophyceae. Teil 1 Naviculaceae, 876 p. Teil 2 Bacillariaceae, Epithemiaceae, Surirellaceae, 596 p. Teil 3 centrales, Fragilariaceae, Eunotiaceae, 576 p. Teil 4 Achnanthaceae, 437 p.

Lafont M., Coste M., Wasson J.G. \& Faessel B. 1988. Comparaison de quatre indices biologiques pour apprécier l'impact de la pollution dans des cours d'eau français. Naturalist can. (Rev. Ecol. Syst.) 115 ; 77-87.

Lange-Bertalot H. 1979. - Pollution tolerance of diatoms as a criterion for water quality estimation. Nova Hedwigia 64 : 285-304.

Leclercq L. 1988. - Utilisation de trois indices, chimique, biologique et biocénotique pourl'évaluation de la qualité de l'eau de la Joncquière, rivière calcaire polluée par le village de Doische (Belgique, prov. Namur). Mem. Soc. Roy. Bot. Belg. 10 : 26-34.

Leclercq L. 1989. - Intérêt et limites des méthodes d'estimation de la qualité des eaux courantes. Rapport complémentaire au cycle de cours spécialisés en épuration des eaux dans les zones rurales. Arlon, 29 avril-24 juin 1988: $47 \mathrm{p}$.

Leclercq L. \& Depiereux E. 1987. - Typologie des rivières oligotrophes du massif ardennais (Belgique) par analyse multivariée de relevés de diatomées benthiques. Hydrobiologia, 153 : 175-192.

Leclercq L. \& Maquet B. 1987. - Deux nouveaux indices chimique et diatomique de qualité d'eau courante. Application au Samson et à ses affluents (bassin de la Meuse belge). Comparaison avec d'autres indices chimiques, biocénotiques et diatomiques. Inst. Roy. Sci. Nat. Belgique, doc trav. 28, 113 pp.

Leclercq L. \& Vandevenne L. 1987. - Impact d'un rejet d'eau chargée en sel et d'une pollution organique sur les peuplements de diatomées de la Gander (Grand-Duché de Luxembourg). Cah. Biol. Mar. 28 : 311-317.

Lecointe C., Coste M. \& Prygiel J. 1993. — OMNIDIA » : a software for taxonomy, calculation of diatom indices and inventories management. Hydrobiologia, 269/270 : 509-513.

Legendre L. \& Legendre P. 1984. - Ecologie numérique. T 1 : le traitement multiple des données écologiques; T 2 : la structure des données écologiques. Coll. Ecologie, $2^{\mathrm{e}}$ ed., $\mathrm{n}^{\circ} 13$ et 14, Masson, 260 et $235 \mathrm{p}$.

Mann D.G. 1989. - The diatom genus Sellaphora : separation from Navicula. Br. Phycol: J. 24 : 1-20.

Newman P.J. 1988. - Classification of surface water quality. Heinemann Professional Publishing, 189 p.

Patrick R. 1949. - A proposed biological measure of stream ; conditions based on a survey of the Conestoga basin, Lancaster country, Pennsylvania. Proc. Acad. nat. Sci. Philad. 101 : 277-341.

Prygiel J. 1991. - Use of benthic diatoms in surveillance of the Artois-Picardie basin hydrobiological quality. In Whitton B.A.W, Rott E. \& Friedrich G. (ed.), Use of algae for monitoring rivers, Düsseldorf, E. Rott, Innsbruck : 89-96.

Prygiel J. \& Coste M. 1993. - The assessment of water quality in the Artois-Picardie water basin (France) by the use of diatom indices. Hydrobiologia, 269/270 : 343-349.
Rott E. 1991. - Methodological aspects and perspectives in the of periphyton for monitoring and protecting rivers. In Whitton B.A., Rott E. \& Friedrich G. (ed.), Use of algae for monitoring rivers, Düsseldorf, E. Rott, Innsbruck : 9-16.

Round F.E. 1991. - Diatoms in river water-monitoring studies. J. Appl. Phycol. 3 : 129-145.

Round F.E., Crawford R. \& Mann D. 1990. - The diatoms. Biology and morphology of the genera. Cambridge univ. Press (ed.), $747 \mathrm{p}$.

Rumeau A. \& Coste M. 1988. - Initiation à la systématique des diatomées d'eau douce pour l'utilisation pratique d'un indice diatomique générique. Bull. Fr. Pêche Piscic. 309 : 1-69.

Sabater S., Sabater F. \& Tomas X. 1987. - Water quality and diatom communities in two catalan rivers. Wat. Res. 21,8 : 901-911.

Sabater S., Sabater F. \& Armengol J. 1988. - Relationships between diatom assemblages and physico-chemical variables in the river Ter (NE Spain). Int. Rev. Ges. Hydrobiol. 73 : 171-179.

Scherrer B. 1984. - Biostatistiques. Gaetan Morin (Ed.), Chicoutimi, Québec, Canada, 850 p.

Schoeman F.R. \& Haworth E.Y. 1986. - Diatoms as indicators of pollution. Report on a workshop. In M. Ricard (ed.), Proc. of the 8th. Internat. Diatom Symp. Paris Aug. 84, O. Koeltz Publ., Koenigstein. : 757-759.

Sladeček V. 1986. - Diatoms as indicators of organic pollution. Acta Hydrochim. Hydrobiol. (14/5) : 555-566.

Sullivan M. 1986. - Mathematical expression of diatom results : are these pollution indices valid and useful ? In M. Ricard (ed.), Proc. of the 8th Internat. Diatom Symp. Paris 1984, O. Koeltz publ., Koenigstein, 772-776.

Van Dam H. 1982. - On the use of measures of structure and diversity in applied diatom ecology. Nova Hedwigia 73 : 97-115.

Steinberg C. \& Schiefele S. 1988. - Biological indication of trophy and pollution of running waters. $Z$. Wasser-Abwasser-Forsch. $21: 227-234$.

Vanlandingham S.L. 1976. - Comparative evaluation of water quality of the St.Joseph river (Michigan and Indiana, USA) by three methods of algal analysis. Hydrobiologia, 48, 2 : 145-173.

Verneaux J. \& Tuffery G. 1967. - Une méthode zoologique pratique de détermination de la qualité des eaux courantes. Indices biotiques. Ann. Sci. Univ. Besançon 3 : 79-89.

Verneaux J., Galmiche P., Janier \& Monnot A. 1982. - Une nouvelle méthode pratique d'évaluation de la qualité des eaux courantes. Un indice biologique de qualité générale (I.B.G.). Ann. Sci. Univ. Fr. Comté, Besançon, Biol. Anim. 4 (3) : 19 p.

Whitton B.A., Rott E. \& Friedrich G. 1991. - Use of algae for monitoring rivers. Proc. Internat. Symp. Düsseldorf, Germany 26-28 May 1991, Innsbrück : 193 p.

Woodiwiss F.S. 1964. - The biological system of stream classification used in the Trent River Board. Chemistry and industry : 443-447.

Wright J.F., Armitage M.T., Furse M.T. \& Moss D. 1988. - A new approach to the biological surveillance of river quality using macroinvertebrates. Verh. Internat. Verein. Limnol. 23 : 1548-1552.

Zelinka M. \& Marvan P. 1961. - Zur Prasisierung der biologischen Klassifikation des Rheinheit fliessender Gewässer. Arch. Hydrobiol. 57 : 389-407. 